\title{
The warm inflationary universe
}

\author{
Arjun Berera ${ }^{1}$ \\ 1 School of Physics, University of Edinburgh, Edinburgh, EH9 3JZ, United Kingdom
}

\begin{abstract}
In the past decade, the importance of dissipation and fluctuation to inflationary dynamics has been realized and has led to a new picture of inflation called warm inflation. Although these phenomena are common to condensed matter systems, for inflation models their importance has only recently started to be appreciated. The article describes the motivation for these phenomenon during inflation and then examines their origins from first principles quantum field theory treatments of inflation models. Cosmology today is a data intensive field and this is driving theory to greater precision and predictability. This opens the possibility to consider tests for detecting observational signatures of dissipative processes, which will be discussed. In addition it will be discussed how particle physics and cosmology are now working in tandem to push the boundaries of our knowledge about fundamental physics.
\end{abstract}

PACS numbers: 98.80.Cq, 05.70.Ln, 11.10.Wx

Published in Contemporary Physics 47, 33 (2006).

\section{INTRODUCTION}

By the 70s the hot Big Bang model had proven very successful in explaining both general qualitative features of our Universe, such as its expansion and presence of a background radiation, as well as providing a quantitatively successful theory of nucleosynthesis of the light elements. However there were difficult problems within this model, which in the early 80's were shown could be nicely solved through the inflation idea. Since its introduction, inflation has been a theoretically very compelling picture in search of justification from observation. In the past decade, precise data on the cosmic microwave background radiation $(\mathrm{CMB})$ obtained primarily from satellite experiments has provided support for inflation. This success has summoned growing interest both in constructing realistic particle physics motivated models of inflation and in calculating predictions from these models as accurately as possible. One outcome that has emerged from examining inflation models at close scrutiny has been that dissipative and nonequilibrium effects during the inflation phase are prevalent in many such models. This has led to an understanding that there are two distinct dynamical realizations of inflation, which have been termed cold and warm inflation. Cold inflation coincides with the original inflation picture and has been the subject of many reviews [1]. The purpose of this paper is to explain warm inflation and review progress in developing this idea.

Warm inflation is not a radical rethink of inflation, but rather a completion of the original picture. The idea stems from an elementary observation. The central theme of inflationary dynamics today is the evolution of a scalar field, which during inflation carries most of the energy of the universe and which interacts with other fields. On the one hand, the standard inflation picture makes a tacit assumption that these interactions have no other effect except to modify the scalar field effective potential through quantum corrections. On the other hand, the warm inflation picture is that interactions not only modify the scalar field potential but also lead to fluctuation and dissipation effects. Certainly in condensed matter systems the effect of interactions generally lead to all three of these effects (several examples are given in [2]). Also from a statistical mechanics perspective the scalar field would want to dissipate its energy to other fields and the whole system would try to equally distribute the available energy. Ultimately the question must be answered through a thorough dynamical calculation.

Dynamics in cosmology differs in one important way from condensed matter systems, in that all processes occur in the presence of an expanding universe. Expansion acts like an external force which is constantly altering the state of any cosmological scale system. For example energy in the universe is continually being diluted due to expansion. Similarly, the configuration of any cosmological scale process is being altered over time. Thus if the quantum mechanical processes that lead to dissipation operate at a time scale much slower than the expansion rate of the universe, than these processes could be totally shut down due to expansion, even if the same processes in a nonexpanding system, like a condensed matter system, operates efficiently. This is the important question that must be understood.

The problem being posed here may appear very specialized and technical. Quite oppositely, what makes the problem interesting is that it combines simple physics from very different disciplines, i.e. cosmology, particle physics and condensed matter physics. The challenge is in appreciating how these different ideas join together, and that is what is to be explained. The other reason this problem is interesting, which also will be discussed in this article, 
is that the consequences of dissipation and fluctuation during inflation have distinct model building implications for particle physics that do not arise in cold inflationary dynamics, where these effects are negligible. This article is fully self-contained, starting from the relevant background in General Relativity and inflation before addressing the main topic. For the reader familiar with these preliminaries, they can skip the next four sections and start immediately from Sect. VI.

\section{BASIC EQUATIONS OF COSMOLOGY}

The single most significant fact about our universe is that all galaxies, except a few nearby ones, are receding from us. Moreover, the redshift, a measure of the recession speed, is larger for the fainter more distant galaxies. This suggests that our universe is expanding, with clusters of galaxies getting more widely separated, and so more thinly distributed through space, as time goes on.

To understand this expansion, or any other aspect of the dynamics of the universe, Einstein's General Theory of Relativity must be used. In General Relativity, matter and geometry are intrinsically entangled. General Relativity treats gravity as a deformation in the geometry of space and in the flow of time, due to the presence of matter. An analogy would be to represent space by a stretched membrane. For a very light ball rolling on it, the surface would be flat. Now imagine placing a heavy ball on this membrane. Its effect would be to depress the membrane in its vicinity. The light ball now would no longer roll in a straight line, but would curve in the direction of the heavy ball. The depression in the membrane is analogous to gravity which is produced due to the presence of matter, in this case the heavy ball. So just as in Einstein's picture, the acceleration of the light ball due to the presence of gravity is viewed as arising due to a deformation in the geometry of space.

The geometry of the universe is expressed through the metric tensor $g_{\mu \nu}$ whereas the matter is described through the energy-momentum tensor $T_{\mu \nu}$. The geometry and matter are related through the Einstein equation

$$
R_{\mu \nu}-\frac{1}{2} g_{\mu \nu} R=-8 \pi G T_{\mu \nu}
$$

where $G$ is the Newton constant of gravitation, $R_{\mu \nu}$ is the Ricci tensor, and $R \equiv g_{\mu \nu} R^{\mu \nu}$ is the curvature. The Ricci tensor is constructed from various derivatives of the metric tensor $g_{\mu \nu}$ and gives a measure of the curvature of space-time. The Einstein equation (1), as can be seen, is an equation relating tensors, here four by four matrices, and so in general there are 16 separate equations. All gravitational phenomenon are governed by these equations.

Our interest is cosmology, meaning phenomena which in each epoch occurs on time-scales of order the age of the universe at that time and on distance scales as large as the size of the universe at the given time. On these cosmological scales, the foundations of the whole subject is embodied in the cosmological principle, which states no observer occupies a preferred position in the universe. This principle implies the universe must be homogeneous (looks the same from every point) and isotropic (looks the same in all directions). Note that homogeniety does not automatically imply isotropy, with a simple counterexample being a universe with a uniform magnetic field; in such a case, every point looks the same as every other, but there is a preferred direction. However isotropy about every point does imply homogeniety, giving a universe in which matter is smoothly distributed.

To quantify the cosmological principle, in regards the geometry, the metric of a space-time which is spatially homogeneous and isotropic is given by the Friedmann-Robertson-Walker (FRW) metric,

$$
d s^{2}=d t^{2}-a^{2}(t)\left[\frac{d r^{2}}{1-k r^{2}}+r^{2} d \theta^{2}+r^{2} \sin ^{2} \theta d \beta^{2}\right]
$$

where $(r, \theta, \beta)$ are spherical-polar coordinates parametrizing the spatial dimensions, $t$ is cosmic time, and $k=1,-1,0$ describe spaces of constant positive, negative and zero spatial curvature. The most important quantity here is $a(t)$ which is the cosmic scale factor and describes the expansion of the universe.

For the metric Eq. (2), the Einstein equations Eq. (1) reduce into two independent equations, one the celebrated Friedmann Equation

$$
\frac{\dot{a}^{2}}{a^{2}}+\frac{k}{a^{2}}=\frac{8 \pi G}{3} \rho,
$$

and the other an equation no one ever bothered to name

$$
2 \frac{\ddot{a}}{a}+\frac{\dot{a}^{2}}{a^{2}}+\frac{k}{a^{2}}=-8 \pi G p .
$$


The expansion rate of the universe is determined by the Hubble parameter $H \equiv \dot{a} / a$, which is the single most important parameter characterizing the universe. The Hubble time $H^{-1}$ sets the time scale for expansion, with the scale factor $a$ doubling in approximately one Hubble time. This fact also means the Hubble length $c / H$ sets the scale of the observable universe, meaning the distance a light beam has traversed during the age of the universe. The Hubble parameter today $H_{0}$, relates the velocity $v$ of the distant galaxies receding away from us to their distance, $r_{0}$, from us via the Hubble law $v=H_{0} r_{0}$.

The matter content in the FRW model is described through just two quantities, the energy $\rho$ and pressure $p$ densities, which in the simplest models are related through the equation of state $p=w \rho$. Given an equation of state $w$, one can then solve the cosmological Einstein equations (3) and (4). Three cases are of particular importance for describing the cosmology of our universe

$$
\begin{aligned}
\text { radiation } & =\frac{1}{3} \rho \Rightarrow \rho \propto a^{-4}, \quad a \propto t^{1 / 2}, \\
\text { matter } & p=0 \Rightarrow \rho \propto a^{-3}, \quad a \propto t^{2 / 3} \\
\text { vacuum } & p=-\rho \Rightarrow \rho \propto \text { const., } \quad \ln (a) \propto t .
\end{aligned}
$$

One comment here on terminology. The vacuum energy is also often referred to as the cosmological constant, with particle physicists preferring the former and General Relativists the latter.

\section{STANDARD COSMOLOGY}

What is called the Hot Big Bang model, and also referred to as the Standard Cosmological Model, is characterized by a universe in which large scale gravitational attraction causes the universe to expanding at a decelerating rate $\ddot{a}<0$. In particular, the Big Bang model is comprised of the first two solutions from Eq. (5), along with some general knowledge about the particle content of the universe and some basic statistical mechanics. Based on this information, the Big Bang model asserts that the late phases of the universe right up to the present are in a matter dominated era, which means a period when the evolution of the scale factor goes as the matter case in Eq. (5) (there is evidence that in the very recent epoch the universe might be in a regime dominated by vacuum energy. This detail is not relevant to our discussion and so we will not delve into it. There are several reviews on this subject [3, [4]). The expansion of the universe implies the earlier universe gets increasingly denser and hotter. Going back in time, the Big Bang model finds that at some stage in the past the universe was in a radiation dominated regime which characterizes the early phase of the universe.

Since the expansion implies the younger universe was smaller and hotter, based on our current knowledge of physics, this leads to a picture of the universe when it was only a few minutes old at at a temperature of $10^{10} \mathrm{~K}$. The significant point is this picture can be tested. One of the most important tests is that the light elements are predicted to be 'cooked' at this time, a process called Big Bang nucleosynthesis (BBN). Thus we can compare calculations of the cosmological fraction of elements like hydrogen, helium, deuterium and lithium with their observed abundances. For example the visible matter content in the universe is found to be about $23 \%$ helium, and this is in excellent agreement with predictions from the Big Bang model. This result is a more stringent verification of the Big Bang model than what might meet the eye. For example it may have happened that astronomers had found astrophysical scaled objects whose helium abundance was far below this prediction of the Big Bang model. This would have been fatal, since extra helium made in stars could boost helium above its pregalatic abundance, but there appears to be no way of converting all helium back into hydrogen. Another verification of the Big Bang model, from nucleosythesis in the early universe is deuterium (heavy hydrogen) abundance. There is only a tiny trace of deuterium in the universe, its abundance ratio to hydrogen is about 1/50,000 and this is consistent with predictions from the Big Bang model.

Another robust prediction of the Big Bang model is the CMB. In the past decade the CMB has been very accurately measured in particular by two satellite experiments, the Cosmic Background Explorer (COBE) and the Wilkinson Microwave Anisotropy Probe (WMAP) (for reviews see [5, [6]). These experiments have found the CMB to have a nearly perfect blackbody spectrum at temperature $T_{0}=2.725 \mathrm{~K}$. This result is another stringent test of the Big Bang model. For example it may have happened that the observed CMB spectrum differed too drastically from the expected blackbody form. Also, there are tiny fluctuations in the CMB temperature distribution at one part in $10^{5}$. The Big Bang model predicts certain correlation between fluctuations in the CMB spectrum and matter fluctuations in the universe. It could have happened, that in fact these correlations were not found. The failure of either of these two tests could have been a major blow to the Big Bang model. However every time the data has shown consistency with the model. 


\section{COSMOLOGICAL PUZZLES}

In short, the Big Bang model is amazingly successful. It provides a testable and consistent account of the early universe, which has been verified at least since the era of Big Bang nucleosynthesis $(t \sim 1$ sec $)$ up to the present. Most importantly there is no observational nor experimental data that casts any serious doubts on this model. Nevertheless the model does not explain everything. There are some serious shortcomings to this model, which are often referred to as the cosmological puzzles. These puzzles are not inconsistencies within the Big Bang model, but rather they are questions that the model itself allows to be asked, but within its limits can not answer.

The first and foremost puzzle is that the universe is homogeneous on a much bigger scale than the Big Bang model would predict. This fact is seen most evidently in the CMB. The CMB radiation that we receive from all directions in the sky was emitted about $10^{6}$ years after the birth of the universe. Based on this fact, it would mean for example that the CMB photons in Fig. 1 received today from opposite directions in the sky were separated by about $10^{7}$ light years at time of emission. Thus they could not have had any causal connection with each other. In this case how did they know to be at $2.725 \mathrm{~K}$ temperature within parts in $10^{5}$ ? This is often called the horizon problem.

Closely related to the horizon problem is the formation of structure problem. Observations indicate nonrandom correlations in the distribution of galaxies and clusters of galaxies on scales much larger than 50 Mpc. Beyond this scale, it is very difficult to see how such correlations could have been created in Standard Cosmology. Similarly, the CMB spectrum of fluctuations at time of last scattering is found to be almost scale invariant up to the largest observable scales in the universe $\sim 3000 \mathrm{Mpc}$. In the Standard Cosmology, no causal mechanism can explain this.

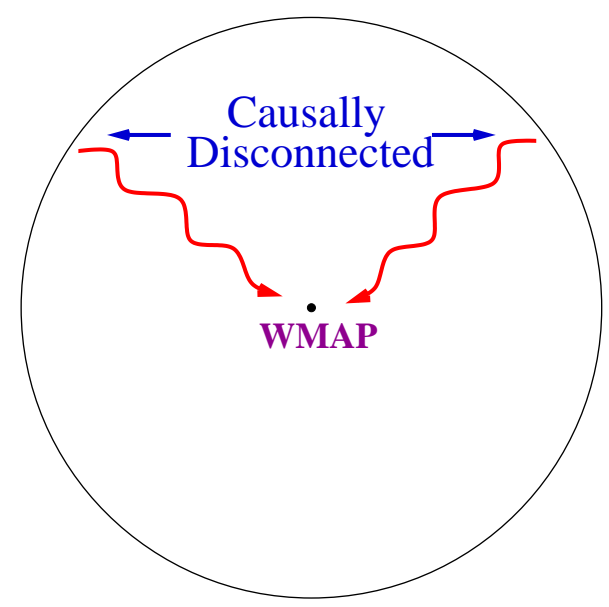

FIG. 1: CMB photons observed from very different directions in the sky would never have been in causal contact according to the Big Bang model

The other major puzzle has to do with the global geometry of the universe. Below Eq. (2) we briefly touched on the fact that in the FRW model, the universe can have a global geometry that is either open, flat or closed. What does that mean? If the universe is flat. than it is described by Euclidean geometry, so two parallel lines would never cross. However the universe may also be open, in which case parallel lines diverge from one another, or it may be closed, like the way the surface of a sphere is closed, so that parallel lines cross one another like the meridian lines on a globe. What is found from observation is that our universe today is very close to being flat. It has a radius of curvature of order the scale of the observed universe $\sim 10^{28} \mathrm{~cm}$.

Based on General Relativity and the Big Bang model, one can ask, what sort of global geometry should we expect. In GR the only natural length scale is the Planck Length, $l_{p} \sim 10^{-33} \mathrm{~cm}$. Thus one might expect the typical radius of curvature to be of order this scale, but that falls some 60 orders of magnitude below observation. A more useful approach is to solve the cosmological Einstein equations (3) and (4), which should tell us given an initial condition at some early stage of the universe, what the geometry of the universe is today. What one finds is that in the Big Bang model, for either matter of radiation dominated cases, the flat geometry is a repulsive point. This means whatever initial conditions one chooses, whether for a geometry that is open of closed, as time evolves, the universe gets further and further away from the flat geometry, i.e. a open universe becomes more open and a closed one more closed. So the only way the Big Bang model could explain the present near flatness of our universe is if in its early phases the universe was tuned to be very very flat. Typically in physics whenever an extreme tuning is necessary, the usual 
assumption is that some aspect of the problem has been understood incompletely. This problem is often referred to as the flatness problem.

As mentioned in the beginning of this section, one now can see that none of the problems mentioned above imply the Big Bang model is flawed, but rather that it is potentially incomplete. One could simply argue that the initial conditions of the universe were such that the temperature happened to be the same in all the causally disconnected regimes we observe today and the universe in its early phases just happened to be very very close to flat. The problem is these are fine tuning argument. In such a circumstance, one is compelled to ask whether there is some dynamical mechanism that has not been appreciate which provides a simple explanation for these peculiar features.

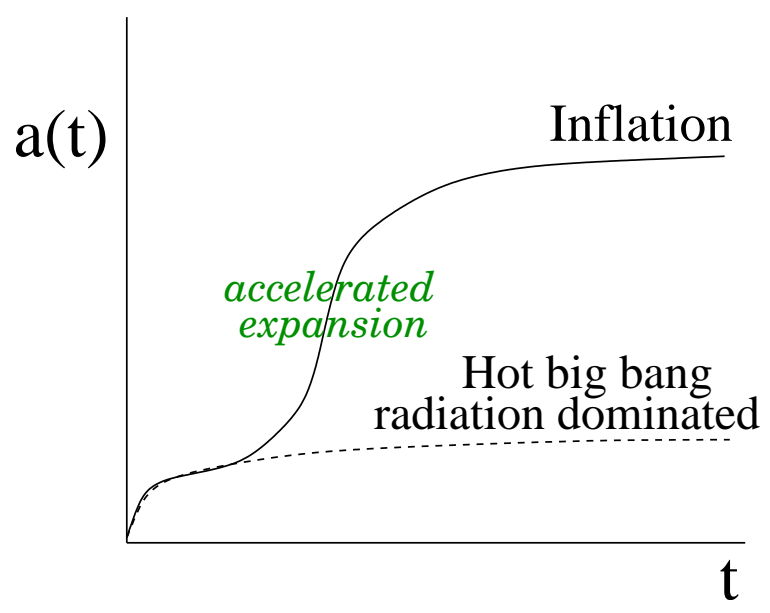

FIG. 2: A schematic representation of how the scale factor in the inflation regime grows much faster than in the Big Bang regime

\section{KINEMATICS OF INFLATION}

As it turns out, one simple and elegant idea, called inflation, can solve both these cosmological puzzles and in a way that nicely fits together with the Big Bang model. The most general description of inflation is that it is a phase in which the scale factor grows at an accelerating rate $\ddot{a}>0$. To study inflation, it is often convenient to use an equivalent form of the cosmological Einstein equations (3) and (4), the scale factor equation

$$
\frac{\ddot{a}}{a}=-\frac{4 \pi G}{3}(\rho+3 p)
$$

and the energy conservation equation

$$
\dot{\rho}=-3 H(\rho+p)
$$

The scale factor equation is obtained by subtracting Eq. (44) from Eq. (3) and the energy conservation equation is obtained by taking the time derivative of Eq, (3) and then use Eq. (4) to eliminate certain terms.

From the scale factor equation it can be seen that in order to obtain an accelerating scale factor $\ddot{a}>0$, it requires $p<-\rho / 3$, thus a substance with negative pressure. This means a universe where the dominant form of matter produces a repulsive form of gravity. The most common example of such a scale factor behavior is exponential, which arises from the "vacuum" case in Eq. (5) and is called a DeSitter space. In simplest terms, a universe undergoing accelerated expansion grows much bigger in the same amount of time as a universe undergoing decelerated expansion. This point is expressed in Fig. 2

This simple idea of having an accelerated growth of the scale factor solves both cosmological puzzles. In the case of the horizon problem, Fig. 3 illustrates the solution. Before addressing this solution, it is important to understand the significance of the time denoted $t_{L S S}$ in this figure. This denotes the time after which photons effectively stop interacting with all forms of matter, an event called "last scattering". Using basic particle physics it is possible to calculate the interaction rates of photons with all other particles, and from that determine quantitively when last 


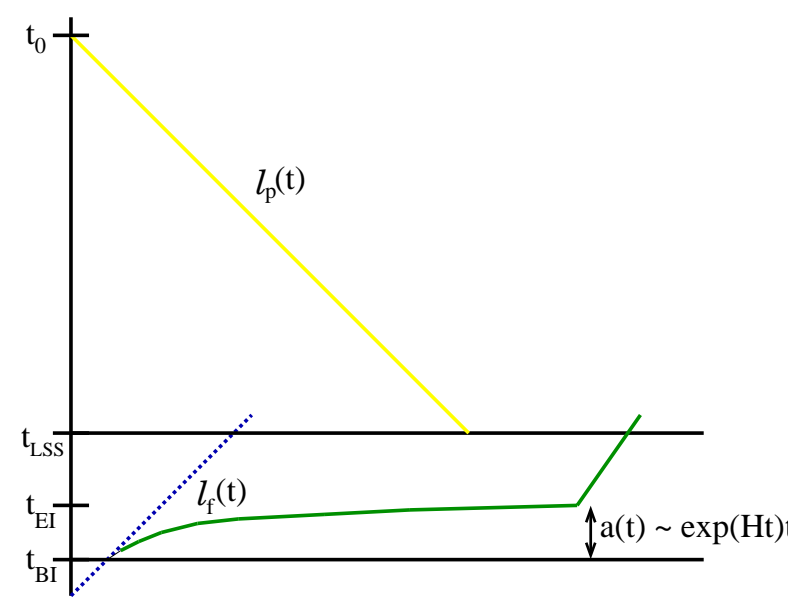

FIG. 3: Lightcone diagram describing the horizon problem and its solution. The lightcone $l_{p}$ (light solid line) shows the maximum region from the past from which an observer today, $t_{0}$, can receive information. The lightcone $l_{f}$ shows the maximum region a light signal can cover since the creation of the universe under Big Bang evolution (dashed line) and under inflationary evolution (heavy solid line).

scattering occurs. This is an important era in the history of the universe. After this time, all the photons in the universe are basically not interacting with anything. This means whatever the distribution of the photon field was at time of last scattering, it remains intact subsequently, aside from simple and calculable effects from the expansion of the universe. Thus, the CMB photon field that is observed today is simply a snapshot of the field at time of last scattering. This is the oldest fossil remnant from the early universe that we have available to us today and that is why there is intense interest in understanding every aspect of the CMB.

Returning to Fig. 3, the dashed line $l_{f}(t)$ represents the forward light-cone of a photon, as it would evolve in the Big Bang model, emitted at the beginning of the universe up to the time $t_{L S S}$. The region to the left of $l_{f}\left(t_{L S S}\right)$ at $t_{L S S}$ represents the region that has been in causal contact at some point in the past before $t_{L S S}$. This is the maximum region that the Big Bang model predicts in which causal processes could for example produce a uniform temperature distribution. The line $l_{p}$ is the past light-cone of an observer today, $t_{0}$. The horizon problem in this context is that in a Big Bang evolution, the region of universe at $t_{L S S}$ from which information is received today is much larger than that which could have been in causal contact at time $t_{L S S}$. In fact simple calculations tell us that there would be something to the extent of $10^{5}$ causally disconnected regions at time of last scattering from which we receive information. This number places into perspective just how starteling it then is that observation finds the CMB temperature basically is the same up to parts in $10^{5}$ from all directions in the sky. The inflation solution to this problem is simply that in such a expansion period, the forward light cone grows much faster. For example for an exponentially growing scale factor, the solid line $l_{f}$ in Fig. 3 illustrates the exponential growth of the forward light-cone, so that at time of last scattering, the region of causal contact incorporates the region within the past lightcone of an observer today, thus solving the horizon problem.

Inflation also solves the flatness problem. The key point being that in a universe undergoing accelerated expansion, the flat geometry becomes an attractive point. For example, for an exponentially expanding scale factor, the universe tends exponentially close to a flat geometry. Thus inflation provides a dynamical mechanism for making the universe extremely flat in its early phases.

Inflation is pictured to occur at an early epoch of the universe, although the time period is not very well pinpointed. In Fig. 4 a time history of the universe is given based on the Big Bang model, with some key events indicated. The success of the Big Bang model since nucleosyntheis (BBN) to the present suggests that the necessary amount of inflation needed to solve the cosmological puzzles could not have occurred during this recent time period. Moreover by combining knowledge from particle physics and cosmology, the time can be pushed back substantially. Supersymmetry (SUSY) is an idea that has an important role to inflation model building. The details of SUSY and its relevance to inflation will be discussed later in the article. Here it is only mentioned that we know SUSY must no longer be a symmetry of nature below the electroweak scale $(T \sim \mathrm{TeV})$, Thus if SUSY is to be useful for inflation model building, than this implies inflation must have occurred somewhere above the $1 \mathrm{TeV}$ scale, which from Fig. 目 means occurred before $\sim 10^{-12}$ seconds after the creation of the universe. 


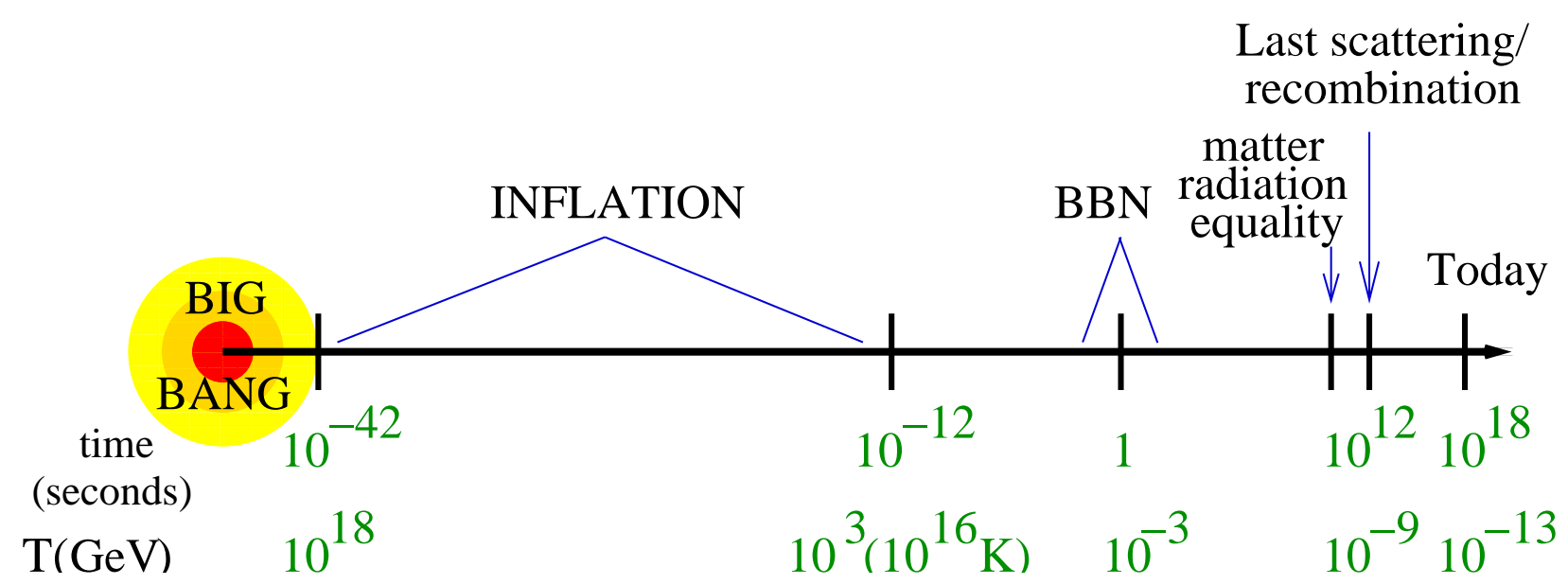

FIG. 4: A time history on the universe

The idea of cosmological repulsion should be credited to Einstein in 1917 who introduced what he called the cosmological constant into his General Relativity equations. His motivation was to allow a static universe in which this repulsive term counteracts the gravitational attractive force arising from the matter in the universe. This idea was dropped after Hubble's discovery that the universe is expanding. Subsequently De Sitter in the mid 1920's built a model involving just a cosmological constant, which meant the universe expanded exponentially forever. In more modern times, in the 70's Gliner was one of the first to recognize the inflation idea [7]. Also in the '70's, the idea of cosmological phase transitions was developed, with major work done by Kirzhnits and Linde [8]. In '79 Starobinsky came up with a model of exponential expansion using quantum gravitational effects and higher derivative curvature terms [9]. The pivotal step was in 1981 with Alan Guth's seminal paper [10] which suggested exploiting a stage of exponential expansion in some supercooled vacuumlike state, that occurs during a first order phase transition, to solve the cosmological puzzles. It was in this paper that the name inflation was dubbed. At about the same time Sato 11] also had studied the effects of a first order phase transition which could provide the necessary conditions for exponential expansion. Guth's paper offered a very clear and elegant idea, but it had the problem of making the universe too inhomogenous after inflation. In 1982 the new inflation scenario was suggested by Linde [12] and Albreacht and Steinhardt [13]. This model set the basic paradigm of scalar field driven inflation and nowadays is regarded as the standard picture of inflation. In the mid-90s it was recognized that since inflation models are interacting dynamical systems, they should exhibit fluctuation and dissipation effects. This observation led to the warm inflation picture [14, 15]. Although warm inflation is just a completion of the original inflation picture, it does alter the basic picture and so it is convenient to treat as a second dynamical realization of inflation.

\section{INFLATION FROM QUANTUM FIELD THEORY}

For theorists, the reason so much excitement and interest centers around inflation is that particle physics contains the basic ingredients necessary to realize inflation. Our most fundamental understanding of nature at present is quantum field theory. In this approach, each particle is represented through a quantum field. The dynamics of the whole system of particles is expressed through a Lagrangian, from which the Lagrange equations of motion for all the quantum fields is obtained. Current quantum field theories of matter contain three types of fields, spin $1 / 2$ fermions, such as the electron, spin 1 gauge bosons, such as photons, and spin zero bosons, such as the Higgs. Although there is ample evidence for the existence of spin $1 / 2$ fermions and spin 1 gauge bosons, no elementary spin 0 scalar particle has yet been detected. Nevertheless, all modern particle models rely on the existence of such fields and the Standard Model Higgs particle is the foremost candidate that particle physicists hope to find at the upcoming Large Hadron Collider (LHC).

From General Relativity we know that in order to realize inflation, it requires an equation of state $p<-\rho / 3$, thus a substance with negative pressure. As it turns out, scalar fields can provide such an equation of state. The energy 
and pressure density of a scalar field is

$$
\begin{aligned}
& \rho=\frac{\dot{\Phi}^{2}}{2}+\mathrm{V}(\Phi)+\frac{(\nabla \Phi)^{2}}{2 a^{2}} \\
& p=\frac{\dot{\Phi}^{2}}{2}-\mathrm{V}(\Phi)-\frac{(\nabla \Phi)^{2}}{6 a^{2}}
\end{aligned}
$$

so that a state which is dominated by the potential energy of a scalar field has a negative pressure. Thus the idea that has generally been adopted in realizing inflation from particle physics is to get the potential energy of some scalar field to dominate the energy density of the universe for some short time period in the early phases of the universe, thereby generating the requisite amount of inflation necessary to solve the cosmological puzzles. Then once enough inflation has occurred, somehow put the universe back into a radiation dominated hot Big Bang regime. The scalar field that performs the task is driving inflation is called the inflaton. There are two essential roles the inflaton must perform. First it must supply an appropriate energy density to be conducive for inflation. Second the fluctuations of the inflaton must have the appropriate features to seed density fluctuations in the universe.

\section{TWO DYNAMICAL REALIZATIONS OF INFLATION}
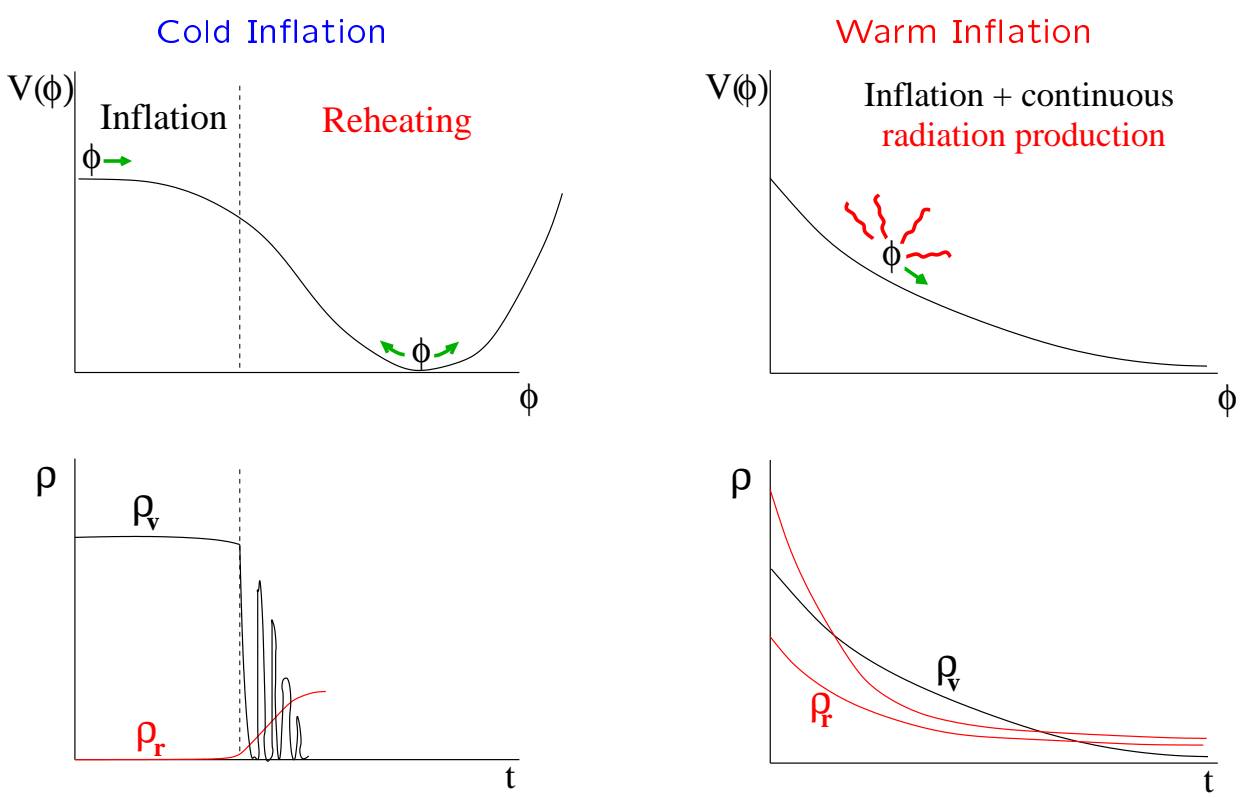

FIG. 5: Comparison of the cold and warm inflationary pictures, top graphs show the scalar field evolution and the bottom graphs show the vacuum and radiation energy density evolution

Inflation is a paradigm, not a theory. Thus, it does not make specific predictions in the same way as the Standard Model of particle physics. Each specific model of inflation makes definite predictions, but the whole class of models can be tested only by looking for generic features that are common to all models. There are two underlying dynamical realizations of inflation, into which all models fall, cold and warm inflation. The basic pictures describing both these dynamics is summarized in Fig. [5 and will be discussed in detail in this section. Cold inflation is synonomous with the standard inflation picture. This picture will be briefly reviewed, since many of its features are also found in the warm inflation picture. After reviewing cold inflation, attention turns to warm inflation. 


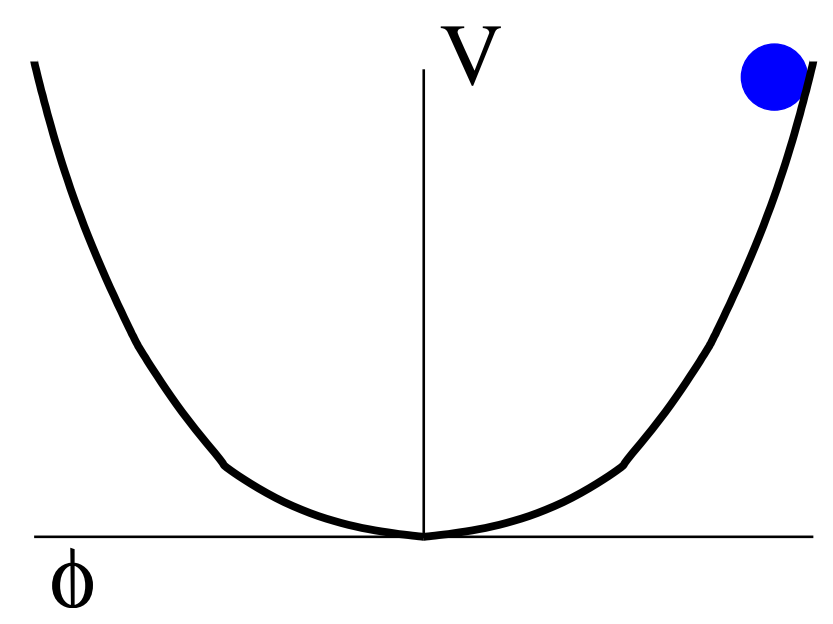

FIG. 6: A typical inflationary potential with the inflaton initially starting at some large amplitude

\section{A. cold inflation}

In the cold inflation picture, the scalar inflaton field is assumed to be essentially in isolation, thus interacting with nothing else besides gravity, during the inflation phase. Also, the scalar field must carry a large potential energy. For example, Fig. 5 shows a symmetry breaking potential for cold inflation, in which when the inflaton is just near the top, $\langle\Phi\rangle \equiv \phi \approx 0$, inflation is expected to occur. For another example, Fig [6]shows a $\lambda \phi^{4}$ potential, in which when the inflaton amplitude is displaced to some $\phi>0$, inflation occurs. The evolution of the scalar field in the FRW universe is described by the General Relativistic version of the Klein-Gordon equation

$$
\ddot{\phi}+3 H \dot{\phi}-\frac{1}{a^{2}(t)} \nabla^{2} \phi-\frac{\partial V}{\partial \phi}=0 .
$$

In this equation the Hubble damping term, $3 H \dot{\phi}$, formally acts like a friction term that damps the inflaton evolution. However this $3 H \dot{\phi}$ term does not lead to dissipative energy production, since its origin is from the coupling of the scalar field with the background FRW metric. As mentioned earlier, the inflaton must fulfill both requirements of driving inflation and providing the seeds for density fluctuations. This section only focuses of the first of these requirements, with the latter taken up in the next section.

In order for inflation to occur, the inflaton must be potential energy dominated, which means the potential energy $V(\phi)$ must be larger than the gradient energy $(\nabla \phi)^{2} / 2$ and the kinetic energy $\dot{\phi}^{2} / 2$. Moreover, in order to obtain enough inflation, these conditions must persist for some span of time. This is often referred to as the "slow roll" conditions. To achieve this, the inflaton field must start out almost homogeneous and almost at rest in some small patch of space. This is possible if the Hubble damping term, $3 H \dot{\phi}$, is large and the potential is very flat. In particular, the slow roll conditions require $9 H^{2}>V^{\prime \prime}$, which if one considers the analogy to the damped harmonic oscillator implies an overdamped evolution for $\phi$, and $V>\dot{\phi}^{2} / 2,(\nabla \phi)^{2} / 2$, so that the potential energy dominates. If these conditions hold in a region of space, then inflation can happen.

For the inflaton dynamics of Eq. (9), it is instructive to see how an inflationary scale factor growth occurs. To a good approximation, the slow roll regime yields an energy density with the vacuum form in Eq. (5). In particular, the only relevant field during inflation in this picture is the inflaton and in the slow roll regime its equation of state from Eq. (8) reduces to $\rho \approx V(\phi) \equiv \rho_{V}$ and $p=-V(\phi)$, with $V(\phi) \approx$ const $\equiv V_{0}$. Applying this to the scale factor equation (6), it leads to $\ddot{a}=8 \pi G V_{0} a / 3>0$, thus an accelerated expansion or in other words inflation. This equation is easily solved to yield $a(t) \propto \exp \left(\sqrt{8 \pi G V_{0} / 3} t\right)$, which is the characteristic exponential growth of inflation described earlier.

The evolution of the energy components in the universe also can be easily studied in this example. During inflation there are two possible components contributing, the vacuum energy density and the radiation energy density. For this system the energy conservation equation becomes $\dot{\rho}_{R}=-4 \sqrt{8 \pi G V_{0} / 3} \rho_{R}$, which has the exponentially decaying solution $\rho_{R} \sim \rho_{R I} \exp \left(-4 \sqrt{8 \pi G V_{0} / 3} t\right)$. In other words, whatever might be the initial radiation energy density in the universe, at the onset of cold inflation this rapidly decays away, thus supercooling the universe. As shown in Fig. 5, 
during cold inflation, the vacuum energy density is large and almost constant, whereas the radiation energy density is negligible.

Once a region of adequately large potential energy materializes, the physics of the subsequent evolution is quite straightforward. The gravitational repulsion caused by the negative pressure drives that region into a period of accelerated expansion. One expresses the amount of inflation as the factor growth in the scale factor at the end of inflation $a_{f}$ to that at the beginning $a_{i}$, and it is usually stated in terms of the efolds $N_{e}$, where $a_{f} / a_{i}=\exp \left(N_{e}\right)$. In order to solve the cosmological puzzles, it is understood that about 60 e-folds of inflation are needed.

Since in this picture the inflaton is completely noninteracting with other matter during inflation, it means in this picture inflation dilutes away any particles that are present at the start of inflation. Thus whatever the temperature of the universe is at the start of inflation $T_{i}$, during inflation it rapidly falls as $T(t)=T_{i} a_{i} / a(t)$. Since the scale factor grows by at least the factor $\exp (60)$, effectively this amounts to a supercooling of the universe, thus the name cold inflation.

Eventually inflation must end and radiation must be introduced into a very cold universe so as to put it back into a radiation dominated Hot Big Bang regime. In the cold inflation picture, the process that performs this task is called reheating [16]. In this process, the Hubble damping term must become small compared to the $\ddot{\phi}$ term in Eq. (9). How this arises can vary from model to model. In Fig. 5 the curvature of the potential becomes very large at some point, which then permits oscillations of the inflaton. In Fig. (6), since $H \sim \phi^{2}$, it means the Hubble damping term will eventually get smaller than the curvature of the potential $V$ as the scalar field falls down far enough. At this point, the inflaton starts oscillating about the origin. In both cases the oscillations cause the inflaton to become kinetic energy dominated, which terminates inflation. If at this point the inflaton is interacting with other matter fields, the oscillations of the inflaton will lead to particle production so that, as shown in Fig. 5, the radiation energy density begins to increase.

\section{B. warm inflation}

The other dynamical realization of inflation is warm inflation. In this picture, similar to cold inflation, the scalar inflaton field must be potential energy dominated to realize inflation. The difference is, in this picture the inflaton is not assumed to be an isolated, noninteracting field during the inflation period. So, rather than the universe supercooling during inflation, such as in the cold inflation picture, instead the universe maintains some amount of radiation during inflation, enough to noticeably alter inflaton dynamics. In particular, the dividing point between warm and cold inflation is roughly at $\rho_{R}^{1 / 4} \approx H$, where $\rho_{R}$ is the radiation energy density present during inflation and $H$ is the Hubble parameter. Thus $\rho_{R}^{1 / 4}>H$ is the warm inflation regime and $\rho_{R}^{1 / 4} \lesssim H$ is the cold inflation regime. This criterion is independent of thermalization, but if such were to occur, one sees this criteria basically amounts to the warm inflation regime corresponding to when $T>H$. This condition is easy to understand since the typical inflaton mass during inflation is $m \approx H$ and so when $T>H$, thermal fluctuations of the inflaton field will become important.

The interaction of the inflaton with other fields implies its effective evolution equation in general will have terms representing dissipation of energy out of the inflaton system and into other particles. A simple phenomenological equation that expresses this is of the Langevin form

$$
\ddot{\phi}+[3 H+\Upsilon] \dot{\phi}-\frac{1}{a^{2}(t)} \nabla^{2} \phi-\frac{\partial V}{\partial \phi}=\zeta .
$$

In this equation, $\Upsilon \dot{\phi}$ is a dissipative term and $\zeta$ is a fluctuating force. Both are effective terms arising due to the interaction of the inflaton with other fields. In general these two terms will be related through a fluctuation-dissipation theorem, but details of that would depend on the statistical state of the system and the microscopic dynamics. In Sect. VIII the origin of these term from first principles dynamics will be examined.

In order for inflation to occur in this picture, the potential energy $V(\phi)$ (vacuum energy density) must be larger than the gradient and kinetic energy, just as in the case of cold inflation. In addition the vacuum energy density must be larger than the radiation energy density $\rho_{R}$. This can be seen from the scale factor equation (6). For a universe filled with vacuum and radiation, this equation becomes $\ddot{a}=8 \pi G a\left(\rho_{V}-\rho_{R}\right) / 3$, so that for an accelerating scale factor it requires $\rho_{V}>\rho_{R}$. From this equation it also can been seen that if $\rho_{R}$ is just a little smaller than $\rho_{V}$, by a factor of two or more, then the scale factor is fairly unaffected by the radiation component and the scale factor growth is almost exponential, just as in the cold inflation case.

The difference from cold inflation is in the evolution of the energy densities, as can be compared in Fig. 5 . In warm inflation the radiation energy does not vanish because vacuum energy is continuously being dissipated at the rate $\dot{\rho}_{V}=$ $-\Upsilon \dot{\phi}^{2}$. The energy conservation equation (7) for this system of vacuum and radiation becomes $\dot{\rho}_{R}=-4 H \rho_{R}+\Upsilon \dot{\phi}^{2}$. 
In this equation the second term acts like a source term which is feeding in radiation energy, whereas the first term is a sink term that is depleting it away. Since the rate of depletion from the sink term is proportional to the amount of radiation present, it means there in general will be some nonzero steady state point for $\rho_{R}$. For example, suppose the source term is just a constant, which is a good approximation during the slow roll evolution of the inflaton, $\Upsilon \dot{\phi}^{2}=$ const. $\equiv c_{0}$. Then the solution to the equation will be $\rho_{R} \approx c_{0} /(4 H)+\left(\rho_{R 0}-c_{0} /(4 H)\right) \exp (-4 H t)$. The second term on the RHS of this solution decays away any initial radiation. However at large time radiation does not entirely vanish because of the first term on the RHS. Thus at large time, the radiation in the universe becomes independent of initial conditions and depends only on the rate at which the source is producing radiation.

As already mentioned, the scale factor equation shows that the presence of radiation during inflation is perfectly well allowed by the equations of General Relativity, since the only requirement it imposes to realize an inflationary scale factor growth is that the vacuum energy density is the dominant component of energy in the universe. Thus there could still be say a $10 \%$ or $1 \%$ etc... admixture of radiation, in addition to the vacuum energy, and inflation would still happen. This is an important point. To appreciate it, note that there are at least five scales in inflation, the vacuum energy $E_{V} \equiv \rho_{V}^{1 / 4}$, the radiation energy $E_{R} \equiv \rho_{R}^{1 / 4}$, the Hubble scale $H$, the inflaton mass $m \equiv V^{\prime \prime}(\phi)$, and the dissipative coefficient $\Upsilon$. In the cold inflation picture, these five energy scales are related as (i). $E_{V}>E_{R}$, (ii). $H>m$, (iii). $m>E_{R}$, and (iv). $H \gg \Upsilon$. Condition (i) is simply a minimal requirement from General Relativity in order to have inflation. Condition (ii) is necessary to be in the slow roll regime. Condition (iii) implies the universe is in a low-temperature regime where radiation has an insignificant effect on inflaton fluctuations. Finally condition (iv) implies dissipative effects have an insignificant effect on inflaton evolution.

Turning to warm inflation, there are two types of regimes that must be addressed, weak and strong dissipative warm inflation. In both these regimes the following energy scales are the same (i). $E_{V}>E_{R},($ ii). max $(\Upsilon, H)>m$, and (iii). $E_{R}>m$. Condition (i) is again required by General Relativity to realize inflation. Condition (ii) is the warm inflation equivalent to being in the slow roll regime. Condition (iii) implies the inflaton fluctuations are no longer in a zero temperature state, thus radiation will have nontrivial effects on inflaton dynamics and fluctuations. Finally the last condition, and the one that leads to two regimes of warm inflation is (iv). $\Upsilon>3 H$, strong dissipative warm inflation and (iv). $\Upsilon \leq 3 H$, weak dissipative warm inflation. The notation here is almost self-explanatory, in the strong dissipative regime, the dissipative coefficient $\Upsilon$ controls the damped evolution of the inflaton field and in the weak dissipative regime, the Hubble damping is still the dominant term.

Even though the presence of radiation need not hinder inflationary growth, it can still influence inflaton dynamics. To appreciate this point, an example will be beneficial. Consider an inflation occurring at the Grand Unified Theory scale, which means $V^{1 / 4} \equiv E_{V} \sim 10^{15} \mathrm{GeV}$. In this case the Hubble parameter turns out to be $H^{2} V^{1 / 2} / m_{P} \sim$ $10^{10} \mathrm{GeV}$. For cold inflation and weak dissipative warm inflation, since the Hubble damping term $3 H \dot{\phi}$ must be adequate to produce a slow roll evolution for the inflaton, it also means the inflaton mass $m \sim 10^{9-10} \mathrm{GeV} \lesssim 3 H$. The key point to appreciate here is that there are five orders of magnitude difference here between the vacuum energy scale and the scale of the inflaton mass. Thus there is a huge difference in scales between the energy scale $E_{V}$ driving inflation and the energy scale $m$ governing inflaton dynamics. This means, for example, in order to excite the inflaton fluctuations above their ground state only requires a minuscule amount of vacuum energy dissipated at a level as low as $0.001 \%$. This gives a good indication that dissipative effects during inflation have the possibility to play a noticeable role (several models of warm inflation exploiting these properties exist [17, 18, 19, 20, 21, 22, 23, 24, 25]). Of course this is only an energetic assessment that is suggestive of interesting physics. It remains a question that a proper dynamical calculation must answer. In particular, the universe is expanding rapidly during inflation at a rate characterized by the Hubble parameter $H$. One must determine whether the fundamental dynamics responsible for dissipation occurs at a rate faster than Hubble expansion.

The other difference of warm inflation to cold inflation is how dissipation effects the parameters of the underlying first principles model. This in particular becomes evident in the strong dissipative regime when $\Upsilon \gg 3 H$. To appreciate this point, note that in cold inflation the inflaton motion is damped by only the $3 H \dot{\phi}$ term. Thus in order to have slow roll evolution, it requires that the inflaton mass $\sim \sqrt{V^{\prime \prime}}$, must be less than $3 H$. However in typical quantum field theory models of inflation, it is very difficult to maintain such a tiny inflaton mass, a point which is explained in greater detail in Sect. XI] This problem is often called the " $\eta$-problem" [39]. In contrast, in warm inflation slow roll motion only requires $V^{\prime \prime}<(3 H+\Upsilon)^{2}$, so when $\Upsilon>3 H$ it means the inflaton mass can be bigger than in the cold inflation case, even much bigger. This relaxation on the constraint in the inflaton mass permits much greater freedom in building realistic inflaton models, since this " $\eta$-problem" is comfortably eliminated.

Another model building consequence differing warm inflation to cold inflation relates to the region of the scalar field amplitude $\phi \equiv\langle\Phi\rangle$ in which the inflation occurs. For cold inflation, for the simplest kinds of potentials, which also are the most commonly used, such as $V=\lambda \phi^{4} / 4$ and $V=m^{2} \phi^{2} / 2$, calculations show that the initial inflaton amplitude has to be above the Planck scale $\phi_{i}>m_{p}$. This arises because the Hubble damping term, $3 H \dot{\phi}$, in these models increases with larger field amplitude, so in order to reach a regime in which slow roll occurs for an adequate time to 
yield the desired 60 or so efolds of inflation, it forces these large field amplitudes. However from the perspective of particle physics, and the ultimate goal of building a realistic inflation model, this condition poses a problem which will be discussed in further detail in Sect. XI. The upshot is that it generally forces more complications into the model building, simply to avoid this problem. On the other hand in warm inflation, when $\Upsilon>3 H$, the added dissipation means the time period of slow roll necessary to obtain the desired 60 or so efolds can be achieved with the inflaton traversing over a much smaller region of its amplitude, thus allowing its amplitude to be smaller. Although the exact answer requires calculation, what is found is that for these simple monomial potentials, for warm inflation the inflaton amplitude is below the Planck scale $\phi<m_{p}$, which simplifies model building as will be further discussed in Sect. XI.

\section{DENSITY PERTURBATIONS}

Zeldovich once likened the universe to an elephant, indeed [26]. His analogy was that globally the shape of the universe may be something very odd, even that of an elephant. Our observable universe in this analogy would be some small part of the elephant's surface, which would look reasonably flat, because its just a small local region. Moreover, in this local region of the elephant's surface, his skin would have wrinkles, and these would be analogous to the perturbations in our universe that emerge as planets, stars, galaxies etc...

As already discussed inflation can explain the origin of homogeniety, but what about the small inhomogenieties in energy density that the universe has. Moreover, as pointed out in Sect. III observation shows that structure in our universe is correlated on scales far too big to be explained by the Standard Cosmology. Inflation has the necessary kinematic features to explain the origin of such structure. To appreciate this point, consider the origin and growth of fluctuations in an expanding universe. At a given epoch of the universe at say cosmic time $t$, causality would imply correlated structure can be produced at that time up to a maximum length scale $\sim c H^{-1}(t)$. Alternatively, if a fluctuation was created at some earlier epoch $t_{e}<t$, and if this fluctuation remains unaltered expect growing due to expansion, then at time $t$ the largest correlated structure to which this could evolve would have a length $\sim a(t) c H^{-1}\left(t_{e}\right) / a\left(t_{e}\right)$. In a Standard Cosmology, meaning $a(t) \sim t^{n}$ with $n<1, c H^{-1} \sim c t$, so that the largest scale of any correlated structure whether produced in that epoch or from an earlier epoch will be $\sim c t$. Consider how these results of Standard Cosmology contrast with those for an inflationary cosmology. In this case the largest possible scale that can be causally produced in a given epoch is $\sim c H^{-1} \approx$ const, whereas if the structure was produced at an earlier epcoh of inflation $t_{e}<t$, then at time $t$ it is correlated on a scale $\approx \exp \left[H\left(t-t_{e}\right)\right] c H^{-1}>c H^{-1}$. In other words, the scale of correlations grows exponentially. Consider the example where a fluctuation is created at the onset of inflation, then after $N_{e}$ efolds of inflation it would have grown by a factor $\exp \left(N_{e}\right)$. Thus if $N_{e} \approx 60$ it means the length scale of the fluctuation has grown by a factor of $\exp (60)$. Contrast this with how much the fluctuation would have grown in the same period of cosmic time in a Hot Big Bang evolution. The 60-efolds of inflation took a time $\sim 60 / \mathrm{H}$. In this time interval the growth of the fluctuation length scale would have only been by a factor of $\approx 60$, so much much less. It is this kinematic feature of inflation which allows production of structure that is correlated on scales much larger than conceivable by causality in a Standard Cosmology.

The kinematic example above implies that provided some dynamical mechanism exists to actually create fluctuations during inflation, the exponential expansion will provide the means to expand those fluctuations to very large scales. Dynamically, the inflaton provides the necessary means for producing density fluctuations during inflation. The key point here is that whereas the homogeneous mode of the inflaton field drives the background cosmology, the small wavelength modes of the field can create the initial density fluctuations. For a quantum field at either zero or finite temperature, it is well known fluctuations of the field will be produced. These fluctuations can be regarded as waves of all possible wavelengths moving in all possible directions. For a field governed by a damped evolution as in Eqs. (9) or (10), the small wavelength modes will oscillate over time, so that the amplitude averaged over a substantial period of time vanishes. However in an exponential expanding universe the situation is different. The wavelengths of all fluctuations of the field grow exponentially along with the expanding universe. When the wavelength of any particular mode becomes larger than the damping scale, $c H^{-1} / 3$ or $c(\Upsilon+3 H)^{-1}$ in Eqs. (9) and (10) respectively, that particular mode stops oscillating and so its amplitude freezes at some particular value. After this time, termed "freeze-out", the amplitude of this mode remains almost unchanged whereas its wavelength keeps growing exponentially. The outcome is the appearance of a classical field that does not vanish after averaging over macroscopic intervals of space and time. This process thereby creates the seeds for structure formation. Over time these initial fluctuations will grow due to gravitational instabilities. The regions of mass overdensity will start attracting more matter to them and those of underdensity will start collapsing, ultimately leading to the clumped formations found in the universe today. Moreover, inflation provides the ingredients for producing the almost scale free spectrum of density fluctuations found in the universe. This arises since slow roll during inflation implies very little changes in the properties of the inflaton field during inflation. Thus each mode that freezes out during inflation will have approximately the same amplitude, thus seeding a near scale free spectrum of density fluctuations. 
The discussion so far is common to both cold and warm inflation. The difference arises in the nature of the fluctuations in these two dynamics. In cold inflation, the inflaton produces zero-point ground state fluctuations. The amplitude of the fluctuations at the point of freeze-out are given by [27]

$$
\delta \phi \sim H
$$

In warm inflation, the inflaton is in some excited statistical state. The fluctuations are induced on the inflaton field by the noise force $\zeta$ in Eq. (10). The most common example, and the only one that will be detailed here is the case where it is in thermal equilibrium at a temperature $T>m$. In this case the amplitude of the fluctuations at freeze-out are

$$
\delta \phi \sim \sqrt{H T}
$$

in the weak dissipative regime [28] and

$$
\delta \phi \sim \sqrt{T(H \Upsilon)^{1 / 2}}
$$

in the strong dissipative regime [29].

The key point is that the nature of the fluctuations in cold versus warm inflation are qualitatively different. This opens up the possibility that observational signatures could be found that might distinguish these two dynamics. This will be discussed further in Sect. XII

\section{FIRST PRINCIPLES DYNAMICS}

It has already been pointed out in Sect VII that even a little conversion of vacuum energy into radiation can have significant effect during inflation. Also the phenomenological equation (10) has been presented as an effective evolution equation for the inflaton field once interactions with other fields are integrated out. The question that remains to be answered from quantum field theory is whether both these effects actually occur and if so in what sorts of models. To address these questions, our task in this section is to start with the fundamental Lagrangian and from that derive the effective equation of motion for the scalar inflaton field.

For any inflaton model the basic Lagrangian quite generally has the form $L=L_{S}+L_{R}+L_{I}$. Here $L_{S}$ is the Lagrangian of the inflaton system itself, which has the general form

$$
\mathcal{L}_{S}=\frac{1}{2} \dot{\Phi}^{2}-(\nabla \Phi)^{2}-V(\Phi)
$$

In any inflation model, this inflaton must interact with other fields, since there must exist channels from which the vacuum energy contained in the inflaton field ultimately can be released into radiation energy in order to end inflation and put the universe back into a Hot Big Bang evolution. These interactions are contained in the $L_{I}$ part of the above Lagrangian. The question is whether this conversion process occurs exclusively at the end of inflation, as pictured in cold inflation, or does it occur concurrent with inflation, as pictured in warm inflation. The most common sorts of interactions would be the inflaton coupled to bosonic fields such as $0.5 g^{2} \Phi^{2} \chi^{2}$ or fermion fields as $h \Phi \bar{\psi} \psi$, i.e. $-L_{I}=0.5 g^{2} \Phi^{2} \chi^{2}+h \Phi \bar{\psi} \psi$. Finally $L_{R}$ contains all other terms associated with all fields aside from the inflaton, like the $\chi$ and $\psi$ fields in this example.

For this Lagrangian, the quantum operator equations of motion can be immediately written down, one for each field. These equations in general are coupled to each other through nonlinear interactions. We are interested in the evolution equation of the fields, or actually their expectation values, given the initial state of the system at some initial time $t_{i}$. For the inflation problem, more specifically we are interested in obtaining the effective equation of motion for the scalar inflaton field configuration $\phi \equiv\langle\Phi\rangle$, after integrating out the $\Phi$ quantum fluctuations, and the effects of all other fields with which $\phi$ interacts, like the $\chi$ and $\psi$ fields in $L_{I}$. This is a typical "system-reservoir" decomposition of the problem, as familiar in statistical mechanics [2]. In our case $\phi$ is the system and all other dynamical degrees of freedom constitute the reservoir.

A simple mechanical model illustrating the system-reservoir procedure is shown in Fig. 7. In this figure the system is the big horizontal spring. This big spring is coupled to a lot of small springs, which comprise the reservoir. The equations of motion for all these springs can be written down. The solutions of the equations for the small reservoir springs can be solved as a function of the big spring coordinate. These solutions can be plugged into the big spring's equation of motion. This procedure in the system-reservoir language is described as integrating out all reservoir degrees of freedom (small springs), to arrive at an effective evolution equation for the system (big spring). 


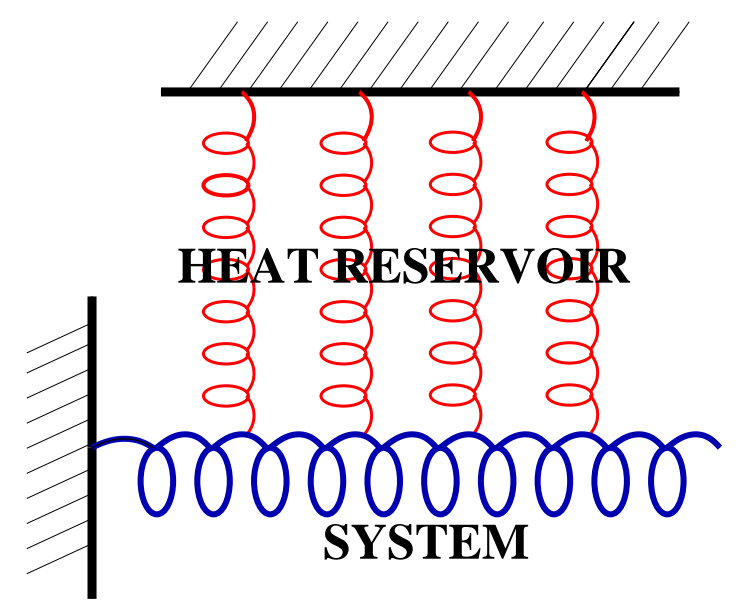

FIG. 7: A mechanical example of a system-reservoir configuration

The system-reservoir approach is applied to many problems in physics. It is instructive to state a few examples here. A very common example is Brownian motion, where one is interested in the evolution of one particle that is singled out, which is immersed in a fluid and interacts with particles in that fluid. One seeks an evolution equation for this Brownian particle, once the effect of all the other particles are integrated out and represented through effective terms in this evolution equation. The analog of the Brownian particle in our problem is the background field $\phi$ and the reservoir bath in our example contains the $\phi$ fluctuation modes, the scalar $\chi$ and the spinor $\psi$. In condensed matter physics, the system-reservoir approach is used in a range of situations, such as for example in the tunneling of a trapped flux in a SQUID, interaction of electrons with polarons in a metal and in Josephson junction arrays [2, 30].

The procedure for obtaining the $\phi$ effective equation of motion is first to replace the field $\Phi$ in the Lagrangian by $\Phi=\phi+\sigma$, where $\langle\Phi\rangle \equiv \phi$ and $\sigma$ are the quantum fluctuations of the $\Phi$ field. The equation of motion for $\phi$, with potential for example $V=m^{2} \Phi^{2} / 2$, then becomes

$$
\ddot{\phi}+3 H \dot{\phi}(t)+m^{2} \phi(t)-\frac{1}{a^{2}(t)} \nabla^{2} \phi+g^{2} \phi\left\langle\chi^{2}\right\rangle+g^{2}\left\langle\sigma \chi^{2}\right\rangle+h\langle\bar{\psi} \psi\rangle=0
$$

In principle what one is trying to do now is solve the quantum operator equations of motion of all the other fields, i.e. $\sigma, \chi$ and $\psi$, as a function of $\phi$, substitute these above in Eq. (15), take the specified expectation values and what would emerge is the sought after effective evolution equation for $\phi$. In practice this procedure can not be done exactly, but various perturbative and resummation methods are used. It is not the purpose of this review to explore these approximation methods, however the interested reader can examine the following references [31, 32, 33]. Here only a few general features of the effective $\phi$ equation of motion are mentioned. First since $\phi$ is isolated, it becomes an open system, so one should expect the $\phi$ effective equation of motion to be nonconservative. Second, the fields $\chi$, $\psi$ etc... at a given time $t_{0}$ in general will be functions of $\phi$ at all earlier times $t<t_{0}$. Thus the expectation values $\left\langle\chi^{2}\right\rangle$ etc... in Eq. (15) will be nonlocal in time with respect to $\phi$, which is consistent with the first general fact, since this will lead to a nonconservative equation.

For the reader familiar with the effective potential in Lagrangian quantum field theory, the origin of the $\phi$ effective equation of motion also can be understood heuristically in another way. An effective potential calculation applies to the situation where $\phi$ is a static background. Its interaction with the other quantum fields leads to the creation of quantum fluctuations which are emitted off $\phi$, propagate in space and time, and then are reabsorbed by $\phi$. These processes are known in the subject as loop corrections, which modify the classical potential and lead to the effective potential. Now suppose $\phi$ is not in a static situation, that it is changing in time. In this case the same loop corrections mentioned above would occur. However at the time of emission and absorption, the state of $\phi$ has changed. Thus these loops no longer simply modify the potential of $\phi$, but also introduce terms which mix products of $\phi$ at different times, thus introducing temporally nonlocal terms into the $\phi$ evolution equation.

The key issue then is, given a particle interaction structure in the Lagrangian, what types of dissipative effects this leads to during inflation. One particular interaction structure has shown to yield very robust radiation production during inflation. This is a two stage mechanism, involving the inflaton field coupled to a heavy scalar field $\chi$ which in turn is coupled to light fermion fields $\psi$ as $g^{2} \Phi^{2} \chi^{2}+h \chi \bar{\psi}^{\chi} \psi^{\chi}$ [34]. In this case the background inflaton field $\phi$ acts 
as a time dependent mass to the $\chi$ field. As $\phi$ changes over time, the $\chi$ mass changes, thus altering the $\chi$ vacuum. This leads to virtual $\chi$ production which then decay into real $\psi$ particles. This type of interaction structure is very common in particle physics models. Thus many models of inflation, which previously were thought to be exclusively cold inflationary are now known also to have regimes of warm inflation [34, 35, 36].

\section{PARTICLE PHYSICS AND COSMOLOGY}

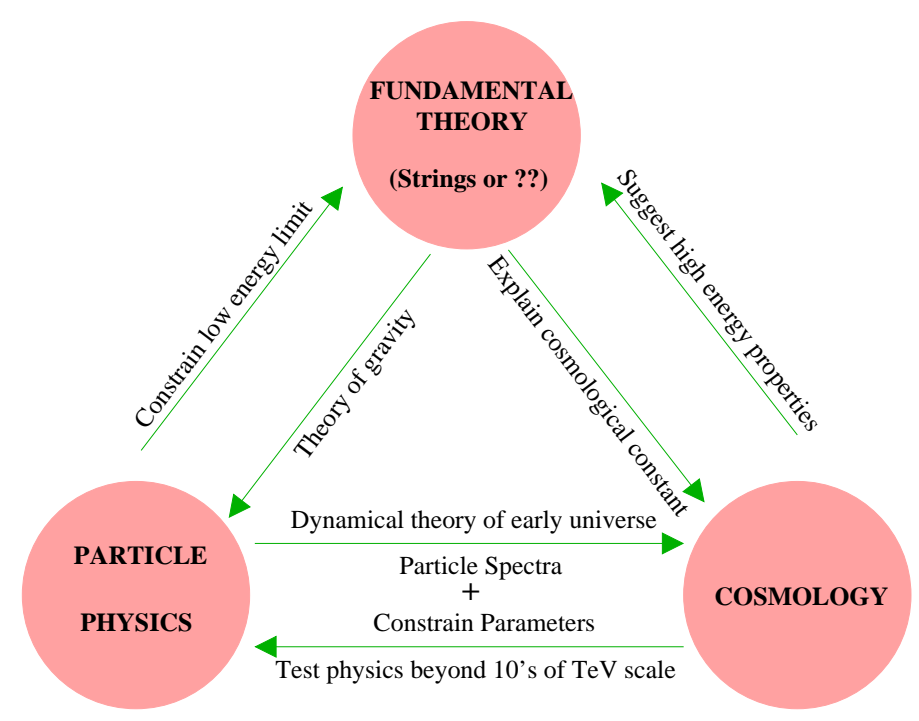

FIG. 8: The three main areas of fundamental physics today. The arrows connecting the three areas show how each field assists and is assisted by the other two.

One might feel that inflation is a nice idea, since it elegantly solves the cosmological puzzles, but because it occurs so far back in the past, what use could it serve physics today. To answer this concern, first some understanding must be developed between the interplay of cosmology and particle physics. At first glance it may appear awkward that there should be any significant connection between cosmology, which treats the largest scales in the universe, and particle physics, which treats the smallest scales. However due to the expansion of the universe, it means in the past the universe was hotter and denser, so that short scale physics becomes increasingly important. This fact has been appreciated since the early days of the Big Bang model. In recent times, the huge increase in precision measurements in cosmology combined with the realization that every $\mathrm{GeV}$ of center-of-mass energy gained at colliders means a long, hard and costly effort, have given impetus to the union of these two disciplines. There are inherent ideological differences between these two subjects. Cosmology is an observational science, not an experimental one. There is just one data sample, our universe. The initial conditions for it have been preset and not only are they not at our disposal to adjust but we also do not know what exactly they were. All information that we can learn about cosmology comes only from what we can observe from the presentday universe. Theory plays the role of providing the basic paradigms within which observations are framed. In contrast particle physics is an experimental science. One can set up a large number of different controlled tests in the laboratory and observe first hand their outcomes. The most basic question that researchers involved in the interface between the two fields are trying to answer is how best to take advantage of the differences to further understanding in both disciplines.

Fig. 8 gives an overview of the state of theoretical particle physics today. The bottom two bubbles represent these two disciplines and show how today they are both active areas of exchange between theory and experiment. The top bubble represents the idealized final theory, that at present is said to be in the process of getting synthesized by the interplay of these two disciplines. The arrows connecting the bubbles represent how each field assists and is assisted by the other two fields. Thus in the case of cosmology, its role to particle physics is to provide a testbed for physics at very high energy scales above the $10 \mathrm{~s}$ of $\mathrm{TeV}$ level, scales too high to be studied in the conceivable future in a man made collider. In return particle physics is providing the means for determining a dynamical theory of the early universe which could be tested by cosmology. 
Falling short of the final theory of everything, one of the more intermediate goals in particle physics is to determine the dynamical theory of the early universe. In particular, the goal is to build a quantum field theory model which reduces to the Standard Model at low energy $E<1 \mathrm{TeV}$ and explains the observed phenomena of particle cosmology at high energy scales. Inflation is the centerpiece of this endeavor. It has already been an excellent example of the interplay between these two disciplines. Realizing inflation from a particle physics models has its challenges. It is not as simple an issue as putting in a scalar field into a particle physics model which has the appropriate features to drive inflation and produce observationally consistent density perturbations. One can build many toy models of inflation that do these two tasks. In coupling with particle physics, additional questions arise such as how does this scalar field interact with other fields in the model and is there any motivation, such as through symmetry, for this field. These questions in turn lead to consequences for the final temperature of the universe at the end of inflation, and how that affects the subsequent Big Bang physics, such as through production of relics, magnetic fields, baryon asymmetry, and defects. Also, the scalar field may lead to other consequences for particle physics at collider scales. Finally, a serious treatment of particle cosmology also must quantify other phenomena such as the baryon asymmetry, the dark matter content, magnetic fields in the universe, and cosmological constant to name an important few. In building such a model, particle physics tends to adopt a minimalist attitude, by trying to restrict the number of free parameters in the model and find relations between different phenomena. One of the ideas of constructing a dynamical model is the hope that different phenomena are found to be interrelated in some way. Thus once one adds all these requirements, the goal of building a QFT model incorporating particle cosmology and particle physics becomes a respectable challenge.

\section{MODEL BUILDING ASPECTS}

The previous section set out the big picture and there are many directions in which that could be developed. Here we return to our discussion of warm inflation and address the special model building features that this dynamics has which distinguish it from cold inflation. Before going into that, first some background into the basic ideas of model building in particle physics is reviewed. The idea of building a particle physics model boils down to choosing a particular collection of spin 0,1/2 and 1 fields, and from these constructing a Lagrangian with some particular choice of interaction terms amongst all the fields. Two guiding concepts play important roles in this construction, symmetry and renormalizibility.

To any transformation acting on the fields that leaves the Lagrangian invariant, there corresponds a symmetry. Such symmetries can be global, meaning they simply shuffle fields amongst themselves, or local, meaning the transformation is different at every point in spacetime. There are also symmetries involving transformations of spacetime. All these symmetries have important impact on an inflation model, in determining the interaction structure of the inflaton field, which in turn determines the effects discussed in previous sections, such as dissipation, fluctuation, radiation content etc... Another important type of symmetry is supersymmetry, which is the idea that the basic laws of nature at the fundamental level are invariant if bosons and fermions are interchanged in the Lagrangian (for a simple review see [37]). Such a symmetry, quite amazingly is consistent with relativistic quantum field theory. In particle physics supersymmetry has been important for solving the hierarchy problem. This problem amounts to the fact that when there are divergent quantum loop corrections, the scale of any dimensional quantity in the theory is not stable. For example, loops introduce divergent quantum corrections to masses. To maintain the scale of these masses then requires fine tuning of parameters at each order in perturbation theory, which is clearly not a meaningful procedure. One of the key features of SUSY is that when one calculates these quantum loops contributing to the masses or any other quantity, the particles and their superpartners both enter the loops. A well known fact of quantum field theory is that the sign of fermion loops is opposite to that of boson loops. Thus for superpartners with the same masses and couplings, which would be the case due to the supersymmetry, these loop corrections would cancel. For this reason, in a supersymmetric theory, the masses and any other quantity can be stable to quantum corrections, This feature of supersymmetry is also important for inflation model building. Density perturbation and slow roll constraints require that the inflaton potential be very flat. If the inflaton is interacting with other fields, then as already mentioned, there will be quantum loop corrections to the inflaton effective potential. However in a SUSY theory these loop corrections cancel to an adequate degree to render the tree-level flatness of the potential to be preserved. For this reason, in a supersymmetric theory, the inflaton potential can be stable to quantum corrections,

The second important element of model building mentioned above was renormalizability. A typical quantum field theory calculation involves the summing up of quantum fluctuations arising from the various fields, i.e. the quantum loops mentioned above. These fluctuations emerge at all energy scales up to some maximum upper bound. If the upper bound is infinity, then it is possible that these summations over quantum fluctuations can be divergent. This is a typical occurrence in quantum field theory calculations, and to treat these infinities, the procedure of renormalization is needed. In this procedure, additional terms are introduced into the Lagrangian, which themselves become infinite as the upper cutoff is removed, but which exactly cancel the infinities from the loop integrals. Depending on the type 
of interactions in the Lagrangian, the number of such counterterms needed to cancel all loop divergences could be finite or infinite.

In the older days, quantum field theory was regarded as a fundamental description of nature at all energy scales. This meant the theory had no upper energy cut-off and so such loop infinities generally would appear. Thus it was argued that the only sensible quantum field theories were those that required only a finite number of counterterms to cancel all loop divergences; such theories have historically been referred to as renormalizable. This condition imposed severe restrictions on the types of interactions in the Lagrangian. For example, fermions could only interact via Yukawa couplings as $\Phi \bar{\psi} \psi$ and scalar fields interaction terms could involve at most a product of four fields. Nowadays Lagrangian quantum field theories are viewed not as fundamental but rather effective theories that are simply low energy approximations to some more fundamental theory such as strings. This modern viewpoint frees the restrictions on the interactions to not just the renormalizable ones, since the Lagrangian theory in any event is only meant to be valid up to some upper energy cutoff.

Collider experiments have convincingly shown that Lagrangian quantum field theories, in particular the Standard Model, is valid at least up to the $1 \mathrm{TeV}$ energy scale. Thus the upper energy cutoff is generally believed to be somewhere above this scale, although where exactly is a matter of differing opinions. The most typical scale is thought to be the Planck scale $m_{P} \approx 10^{19} \mathrm{GeV}$, above which quantum gravity effects are believed to become significant. In the effective theory approach, the relevance of a cutoff means the quantum field theory will have nonrenormalizable interaction terms that can become important above the cutoff scale. Typically very little other information is available, so one must simply write all possible such terms, usually an infinite number of them. For example for a scalar field $\Phi$, these nonrenormalizable terms quite generally would be expressed in a series like $\sum_{n=1}^{\infty} a_{n} \Phi^{4}\left(\Phi / m_{P}\right)^{n}$.

For inflation, these nonrenormalizable interactions would be important when $\langle\Phi\rangle \equiv \phi>m_{P}$. In fact for model building this large $\phi$ amplitude regime is a disaster, due to to the infinite number of unsuppressed terms in the Lagrangian. For this reason, inflation models must be restricted to the regime $\phi<m_{P}$. This is an important point, because, in the simplest sorts of inflationary potentials, meaning monomial potentials like $m^{2} \Phi^{2}$ or $\lambda \Phi^{4}$, as mentioned earlier, in order to achieve adequately large Hubble damping from the $3 H \dot{\phi}$ term, it forces a very large field amplitude which calculations of cold inflation show are in the region of $\phi>m_{P}$ [38, 39]. As such, from a model building perspective, these are not valid models. In contrast, for warm inflationary dynamics in the strong dissipative regime, the increased dissipation allows smaller field amplitudes which calculations show for the simple monomial potentials lead to inflation with $\phi<m_{P}$, thus model building is consistent [29, 36].

Nonrenormalizable interaction terms introduce another possible hurdle for model building. In cold inflation, since the damping term is $3 H \dot{\phi}$ with $H^{2} \sim V / m_{P}^{2}$, in order for slow roll to occur, it means the curvature of the potential must be less than the Hubble scale $V^{\prime \prime}<9 H^{2}$. However supersymmetry models also typically can have nonrenormalizable interaction terms of the form $V \Phi^{2} / m_{P}^{2} \sim H^{2} \Phi^{2}$ [39]. In cold inflation such terms would spoil the slow roll conditions, and so additional constraints on model building are introduced. In contrast, in strong dissipative warm inflation, since the damping term is now $\Upsilon>3 H$, slow roll is possible with much bigger curvature of the potential $\Upsilon^{2}>V^{\prime \prime}>9 H^{2}$ [29, 36]. Thus these sorts of nonrenormalizable interactions have little consequence.

These differences arising from the warm and cold dynamics, lead to significant differences in the model building prospects in the two cases. In general for particle physics models such as the Standard Model and its extensions, at high energy scales it is easy to identify monomial scalar potentials, thus potentials conducive for warm inflation. However, for cold inflation, to overcome the large $\phi$-amplitude problem, the simplest models, known as hybrid models, require two scalar fields coupled in a specific way. Such models do not readily arise in particle physics models but must be added on, thus introducing more parameters and in turn decreasing the predictability of the model.

These model building issues are particularly timely. In two years time the next generation of colliders is expected to be commissioned, the Large Hadron Collider (LHC), which is under construction at the Swiss-French border near Geneva at the European laboratory for particle physics CERN. This machine will accelerate protons to very high energies and then bring them into collision at a center of mass energy of $14 \mathrm{TeV}$. Over a time period spanning beyond the past 10 years, evidence from collider experiments at lower energies to the LHC combined with theory have led to some highly awaited discovery prospects at the LHC. This list starts foremost with the expectation to detect the Higgs boson. Beyond that, or in some quarters maybe instead of that, the most common phrase being touted is finding physics beyond the Standard Model. In particular the top of this list of anticipated outcomes is the discovery of supersymmetry.

SUSY must be a broken symmetry in our world, since we do not observe any of the superpartners. For example, in supersymmetry there is a spin-zero particle with the mass and electric charge of the electron. If such a particle existed many experiments would have already detected it. What we expect is that at sufficiently high energy SUSY becomes unbroken. There is no firm knowledge of what this energy scale is, since in fact as of today there is no firm knowledge that SUSY really has anything to do with nature at all. There are convincing theoretical arguments in favor of its existence. Moreover, Higgs physics and its relation to the masses of the $\mathrm{Z}$ and $\mathrm{W}$ bosons, offer arguments that SUSY may be restored at around the TeV scale. Experiments at Fermilab of protons colliding at center-of-mass 
energy $\sqrt{s}=2 \mathrm{TeV}$ and at the Large Electron Positron Collider LEP at $\sqrt{s}=200 \mathrm{GeV}$ so far have shown no evidence for the existence of SUSY. The LHC will have almost an order of magnitude increase in center-of-mass energy, which offers a significant new testing ground for SUSY.

Particle physicists have constructed extensions to the Standard Model that incorporate SUSY. The simplest such extensions are the Minimal-Supersymmetry-Model (MSSM) and the Next-to-Minimal-Supersymmetry-Model (NMSSM), with the names being self-explanatory. The interesting point for warm inflation is it has been shown that in the NMSSM, the same scalar field responsible for the Higgs mass also can be the inflaton [36]. At large amplitudes the potential for this scalar field has the form $\lambda \Phi^{4}$. Thus cold inflation would not be consistent, due to the large amplitude problem, but warm inflation is possible. Realizing inflation is one of the most difficult hurdles that a model must accomplish in the goal toward building a particle physics model that explains particle cosmology phenomena. Thus, this fact about the NMSSM has opened up the possibility to build perhaps the minimal model that is consistent with the Standard Model at low energy and particle cosmology at high energy. This is a current area of research that is being explored.

\section{TESTING WARM INFLATION WITH OBSERVATION}

In the last decade cosmology has transformed itself from being a data starved to data rich field. The primary data is of the CMB and the source of this data is mainly through the two satellite experiments mentioned earlier COBE and WMAP. These satellites basically have been collecting CMB photons from all directions in the sky over the period of years. From this, a map has been produced of the temperature of the CMB at different locations on the sky, parametrized by the polar angle $\theta$ and azimuthal angle $\beta$. As already mentioned the CMB temperature is almost the same in all direction at $T_{0}=2.725 \mathrm{~K}$ with variations at different points on the sky at the level of one part in $10^{5}$. The most important and detailed information about the early universe is contained in these small temperature fluctuations $\delta T(\theta, \beta)=T(\theta, \beta)-T_{0}$.

For any theoretical model of the early universe, one can calculate its predicted CMB temperature and accompanying fluctuation spectrum. A key meeting point between theory and observation in early universe cosmology is on comparing model predictions against CMB data. In particular, the largest scale fluctuations in the CMB could not have been produced by a causal mechanism in the Standard Cosmology, but as discussed in Sect. VIII inflation offers one of the most compelling explanations for their creation. Moreover each inflation model would predict its own distinct spectrum. Recall from Sect. VIII that during inflation, a given fluctuation mode of the inflaton field will expand along with the universe. Eventually once the wavelength of this mode becomes bigger than the Hubble radius during inflation, this mode freezes after which no causal physics can alter its amplitude. The earlier a mode freezes out during inflation, the bigger the scale of the fluctuation to which it corresponds. In particular, the largest fluctuation scale observed in the universe today corresponds to a fluctuation mode that froze about 60 efolds before the end of inflation. Each subsequent mode that freezes out corresponds to fluctuations in our universe today at successively smaller and smaller length scales. Thus during inflation, each mode that freezes in general will reflect the state of the inflaton field at that time, and this will vary slightly from one mode to the next as they freeze-out. These small variations will differ from model to model, and so each model will show its own characteristic signature in its prediction for the temperature fluctuations of the CMB.

As it turns out, the observed fluctuations in $\delta T(\theta, \beta)$ agree very well with the case where the amplitude of every mode that freezes during inflation is exactly the same, This is known as a scale invariant, or Harrison-Zeldovich, spectrum. The fact that the spectrum produced in scalar field slow roll inflation is almost scale invariant was another of the successes of inflation in the early days. Today, what observers and theorists are looking for is how much the data deviates from scale invariance, and which models of inflation are consistent with these deviations.

Data has shown some interesting types of deviations from scale invariance, although it is still early days in the field for definitive claims. For example, the first year WMAP data indicated the interesting feature that the amplitude of density fluctuations at the largest scales are somewhat suppressed to those at the smallest scales. This is a discriminating feature which could be used to rule out models. In cold inflation, this feature turns out to be very difficult to explain, although models do exist [40]. In contrast, for warm inflation this feature can be explained in some common models, with the important point being that dissipative effects play the key role [41]. Thus if future data supports this first year WMAP finding, it may prove useful in distinguishing the two forms of inflationary dynamics.

There are other interesting features that can arise from dissipative effects. One example is oscillations in the CMB temperature fluctuation amplitude as one varies scales [41]. Also, the nonzero vacuum energy during inflation induces production of gravity waves. The amplitude of gravity waves is proportional to the scale of the vacuum energy, which typically differs in warm versus cold inflation models. So this offers another measurement that could assist in determining the form of dynamics during inflation [42]. The distribution of the fluctuations is another area of investigation. The CMB fluctuations appear to be very close to gaussian distributed, but the small nongaussian 
deviations could provide information that discriminates between warm and cold inflationary dynamics [43].

\section{OUTLOOK}

Inflationary cosmology today is at a crossroad. It has reached the stage where data plausibly argues for the existence of an inflation phase occurring sometime in the early universe. What is unclear at the moment is the level of precision to which inflation can be discriminated. What is clear is no single test will be adequate on its own to firmly establish the existence of inflation. In fact data alone will not be enough. Guidance from quantum field theory also will be necessary to narrow the possible inflation models. The interplay between theory and observation perhaps eventually will even single out just one model.

Warm inflation has played an important role in realizing the prominent role that quantum field theory must play. In the early days where cold inflation was believed to be the only dynamical realization of inflation, the importance of quantum field theory in inflation models was very limited. The inflaton evolution equation was thought of basically as a classical equation with quantum field theory entering only to the extent of the effective potential and the quantum fluctuations of the inflaton. The introduction of warm inflation has shown that interactions during inflation play a much more significant role than just this. The role of dissipation and fluctuation are now seen to be significant features of inflation which in fact could even be the main story during inflation.

[1] Olive, K. A., 1990, Phys. Rep. 190, 307; Lyth, D. H. and Riotto, A., 1999, Phys. Rep. 314, 1.

[2] Weiss, U., 1993, Series in Modern Condensed Matter Physics Vol. 2: Quantum Dissipative Systems, (World Scientific).

[3] Banks, T., 2004, Physics Today 57, 46.

[4] Brax, P., 2004, Contemp. Phys. 45, 227.

[5] Liddle, A. R., 1998, Contemp. Phys. 39, 95.

[6] Peiris, H., 2005, Contemp. Phys. 46, 77.

[7] Gliner, E., 1970, Sov. Phys. Dokl. 15, 559.

[8] Kirzhnits, D. A., 1972, JETP Lett. 15, 745; Kirzhnits, D. A. and Linde, A., 1972, Phys. Lett. B bf 42 , 471.

[9] Starobinsky, A. A., 1980, Phys. Lett. B 90, 99.

[10] Guth, A. H., 1981, Phys. Rev. D 23, 347.

[11] Sato, K., 1981, Phys. Lett. B 99, 66.

[12] Linde, A. D., 1982, Phys. Lett. B 108, 389.

[13] Albrecht, A. and Steinhardt, P. J., 1982, Phys. Rev. Lett. 48, 1220.

[14] Berera, A., 1995, Phys. Rev. Lett. 75, 3218.

[15] Berera, A., 1996, Phys. Rev. D 54, 2519.

[16] Albrecht, A., Steinhardt, P. J., Turner, M. S., and Wilczek, F., 1982, Phys. Rev. Lett. 48, 1437; Dolgov, A. D. and Linde, A. D., 1982, Phys. Lett. B116, 329; Abbott, L. F., Farhi, E., and Wise, M. B., 1982, Phys. Lett. B117, 29.

[17] Berera, A., 1996, Phys. Rev. D55, 3346.

[18] de Oliveira, H. P. and Ramos, R. O., 1998, Phys. Rev. D57, 741.

[19] Gunzig, E., Maartens, R., and Nesteruk, A. V., Class. Quant. Grav. 15, 923.

[20] Lee, W. and Fang. L. Z., 1999, Phys. Rev. D59, 083503.

[21] Maia, J. M. F. and Lima, J. A. S., 1999, Phys. Rev. D60, 101301.

[22] Bellini, M., 1999, Nucl. Phys. 563, 245.

[23] de Oliveira, H. P. and Joras, S. E., 2001, Phys. Rev. D64, 063513.

[24] Chimento, L. P., Jakubi, A. S., Zuccala, N. A., and Pavon, D., 2002, Phys. Rev. D65, 083510.

[25] Brandenberger, R. H. and Yamaguchi, Y., 2003, Phys. Rev. D 68, 023505.

[26] Zeldovich, Y. B., 1988, Physics Today, 41 N3, 27.

[27] Guth, A. H. and Pi, S. Y., 1982, Phys. Rev. Lett. 49, 1110;

[28] Moss, I. G., 1985, Phys. Lett. B 154, 120; Berera, A. and Fang, L. Z., 1995, Phys. Rev. Lett. 74, 1912.

[29] Berera, A., 2000, Nucl. Phys. B 585, 666.

[30] Caldeira, A. O. and Leggett, A. J., 1983, Ann. Phys. 149, 374.

[31] Berera, A., Gleiser, M., and Ramos, R. O., 1998, Phys. Rev. D 58, 123508; 1999, Phys. Rev. Lett. 83, 264.

[32] Berera, A. and Ramos, R. O., 2005, Phys. Rev. D 71, 023513; 2001, Phys. Rev. D 63, 103509.

[33] Lawrie, I. D., 2002, Phys. Rev. D66, 041702.

[34] Berera, A. and Ramos, R. O., 2002, Phys. Lett. B 567, 294; 2005, Phys. Lett. B 607, 1.

[35] Hall, L. M. H. and Moss, I. G., 2005, Phys. Rev. D71, 023514.

[36] Bastero-Gil, M. and Berera, A., 2004, Phys. Rev. D71, 063515; ibid, hep-ph/0507124.

[37] Kane, G. L., 2000, Contemp. Phys. 41, 359.

[38] Linde, A., 1983, Phys. Lett. B129, 177.

[39] Arkani-Hamed, N., Cheng, H. C., Creminelli, P. and Randall, L., 2003, JCAP 0307, 003. 
[40] Linde, A. and Riotto, A., 1997, Phys. Rev. D 56, 1841.

[41] Hall, L. M. H., Moss, I. G. and Berera, A., 2004, Phys. Rev. D 69, 083525; 2004, Phys. Lett. B 589, 1.

[42] Taylor, A. N. and Berera, A., Phys. Rev. D 62, 083517.

[43] Gupta, S., Berera, A., Heavens, A. F., and Matarrese, S., 2002, Phys. Rev. D 66, 043510. 